\title{
The Association between Amino Acid Intake and Polycystic Ovary Syndrome in Women Who Referred to Isfahan University of Medical Science Clinics
}

\author{
Golnaz Rajaeieh ${ }^{1}$, Nafiseh Shokri Mashhadi ${ }^{2}$, Morteza Safavi $^{2}$, Zahra Amini Pozveh $^{3}$, Ali Pezeshki $^{* 1}$ \\ 1-Department of Community Nutrition, National Nutrition and Food Technology Research Institute, Faculty of Nutrition Sciences and Food Technology, \\ Shahid Behehshti University of Medical Sciences, Tehran, Iran \\ 2- Department of Clinical Nutrition/Community Nutrition/Food Science and Technology, Food Security Research Center, School of Nutrition \& Food \\ Science, Isfahan University of Medical Sciences, Isfahan, Iran. Instead of Department of Clinical Nutrition, School of Nutrition and Food Science, \\ Isfahan University of Medical Sciences, Isfahan, Iran \\ 3- Community Medicine Specialist, Isfahan University of Medical Sscience, Isfahan, Iran
}

\section{A B S T R A C T}

Background and Objectives: Polycystic ovary syndrome (PCOS) is the greatest current endocrine disease in generative women. Although other issues such as insulin resistance, hyperinsulinemia, adiposity and dietary pattern are implied to be connected to PCOS, but the most recent evidence are needed to improve management of PCOS. Proteins and amino acids (key dietary components) can also have impact on this condition and may have therapeutic effects for handling this disorder. However, there is little information available in this area. So, the purpose of this study was to assess the connection between amino acids and protein intake and PCOS.

Materials and Methods: Science, Iran. Amino acids and proteins intake was estimated using validated food frequency questionnaire. Other factors such as ovarian disease, inherited predisposition, physical activity, the age of menarche and medical history were assessed by the questionnaire. Data analysis was represented by adjusted logistic by SPSS software version 15 .

Results: The marked association among PCOS and ovarian disease $(P<0.001)$, age $(P<0.001)$ and consuming drugs $(P$ $=0.001)$ were seen. There was inversely associated between body mass index (BMI) and PCOS, but it was not remarkable $(P=0.068)$. But, no statistically significant connection was seen between the use of LEU (Lucien), MET (methionine), CYS (cysteine), PHE (phenylalanine), VAL (valine), ASP (asparagines), GLU (glutamic acid), and PCOS. Although, there was significant relationship between glycine, prolin, and methionine with PCOS.

Conclusions: According to the results of this study, significant relationship was observed between glycine, prolin, and methionine and BCAA/AAA ratio with PCOS.

Keywords: Protein products, Amino acids, Polycystic ovary syndrome

\section{Introduction}

Polycystic Ovary Syndrome (PCOS) is recognized as one of the current problems of endocrine disorders in the women of the generative stages (1-3), affecting $\% 10$ of women $(4,5)$. In fact, this syndrome has been subsequent from the existing of afflicted ovaries of women (6). This dysfunction is responsible for $30-40 \%$ of the current reasons of infertility. In addition, the most leading cause of ovarian disorders is due to PCOS (7).
Owing to the lack of a decisive diagnostic indicator, level of reported prevalence of this syndrome in the various researches is different. The prevalence of PCOS using ultrasound diagnostic method is between $21 \%$ and $22 \%$ (8). On the other hand, in other investigations which use oligomenorrhea and hyperandrogenemia appearance for the diagnostic standards of PCOS, the prevalence level was 9\% (9). 
The exact cause of PCOS is still unknown, nevertheless, some variable, for instance, insulin resistance/hyper insulinaemeia, adiposity, genetic factors, environmental factors, and hormonal factor such as hyper androgenemia, hyperprolactinemia, hypothyroidism or thyroid-related dysfunctions and type of food intake, all may be connected to developing PCOS [10-12]. Besides correlation of PCOS symptoms with those associated with metabolic syndrome, there is some evidence to present PCOS as a pro-inflammatory state and Chronic low-grade inflammation has been suggested as a key contributor of the pathogenesis and development of polycystic ovary syndrome (13 and 14).

Regarding the dietary factors, the intake of some micronutrient were significantly low in women with PCOS [7]. Furthermore, low carbohydrate diet and weight loss worked as anti-inflammatory agents, improving fertility outcomes in PCOS (15 and 16).

Animal products include both saturated and unsaturated fats and some results suggest that a reverse relationship exists between both processed and unprocessed red meat with inflammation markers. However, to our knowledge, there are a few investigations focusing on the relationship between the type of dietary protein and amino acids with PCOS. However, there are some scattered studies evaluating the relationship between protein consumption and PCOS. Chavarro et al, evaluated the association intake of protein from animal and vegetable origin with the risk of ovulatory disorder infertility and revealed that switching animal sources of protein with vegetable sources may decrease ovulatory infertility (17). But, Karakas et al, assessed the dietary intake of protein intake in women with polycystic ovary syndrome compared with the placebo group and found that protein intake did not differ significantly (18). Due to data inconsistency , we aimed to assess the relationship between protein and amino acids intake and risk of polycystic ovary syndrome in women who referred to Isfahan university of Medical Science clinics.

\section{Materials and Methods}

Design of study and participants: This retrospectively observational study was performed in Shahid Beheshti teaching hospital clinic in Isfahan, Iran. The data were collected to evaluate the connection between the values and quality of protein intake and
PCOS condition over the period of March to September 2013. The institutional ethics committee approved the study protocol and written informed consent was signed by the participants. Four hundred women $(n=400)$ were recruited from general practitioner and specialists' hospital clinics by using a simple random sampling.

Due to the importance of inclusion and exclusion criteria for the study, it is worth mentioning that the metrics for the entrance in the study were the desiring to participate, lack of early puberty, lack of uterus cancer or pregnancy. Also, the subjects were prohibited to start study if they did not want to participate or diagnosed with chronic disease.

Evaluation of variables: The data of the food intakes were tested by the researcher using 168 items Food Frequency Questionnaire (FFQ), the questionnaire was explained for the patients and collected consumption information and completed from a given list of foods by them and analyzed by the N4 software. Participants were asked to recall how often, on average, they consumed a given amount of each food during the past single -year period. The amounts were in household or common measures such as 1 slice, 1 tablespoon $(15 \mathrm{~mL})$, or 1 cup $(250 \mathrm{~mL})$, representing 1 standard serve for each food. Response options ranged from "Never" to " $4+$ times a day." The validity and reliability of which were previously confirmed (19). The information on the history of ovary dysfunctions progression, genetics record, primary menstruation age, the background of thyroid diseases, the patients' medication history (any medicine or surgery), the duration of PCOS progression and physical activity were gathered by a nutritionist expert by means of a checklist. Physical activity information was obtained through interviews with the short version of International Physical Activity Questionnaire (IPAQ) and reported as metabolic equivalent (MET).

The weight and height of the participants were examined. The weight $(\mathrm{kg})$ of the subjects were measured with clothes but without shoes with an accuracy of $0.1 \mathrm{~kg}$. The height of participants was calculated without shoes to the nearest $0.1 \mathrm{~cm}$ by a measuring tape. The body mass index was calculated by dividing the weight $(\mathrm{kg})$ value by the square root of height $(\mathrm{m})$ value $\left(\mathrm{kg} / \mathrm{m}^{2}\right)$. The PCOS disorder was recognized by sonography indicative valuation and 
project supervisor checked patients' clinical appearances.

Statistical analysis: Data analysis was done by the SPSS software version 15. Data are shown as mean \pm standard deviation (SD). $P$ value of $\leq 0.05$ was considered significant. Chi-Square Test and Fisher's exact test were suggested for categorical and nominal variables. Student $t$ test was used after using Levene's test for equality of variance to compare participants' age, age at menarche and their BMI. Mann-Whitheny test was used for comparing patients' calorie intake. Random sampling was performed. The power of the study was (1-B) 0.80 , and significance level was $5 \%$ (two-sided). Furthermore, the ratio of PCOS-afflicted patients and the sampling error were expected to be $50 \%$ and $0.07 \%$, so the sample size was identified as many as 400 .

\section{Results}

This study was performed on 400 women administered to the Shahid Beheshti hospital clinics. The mean age of participants was $29.5 \pm 4.8$ years old and the mean of their BMI was $24.1 \pm 4.8$. The prevalence of PCOS in this study was documented to be $9.9 \%$. Based on the preliminary analysis of data (descriptive analysis), as presented in table 1, no differences appeared between PCOS patients and other participants as regards to their age at menarche, BMI, exercise, calorie intake and other disease prevalence such as diabetes and thyroid disease, nevertheless the PCOS patients were considerably younger than others ( $p$ value $=0.008$ ). Additionally, the background of ovarian diseases, genetics and drug use were different between the two groups (table 1). No significant changes were found in physical activities between the two ( $p=0.62$ ).

Table 1. The health condition characteristics of participants

\begin{tabular}{|c|c|c|c|c|}
\hline \multirow[t]{2}{*}{ Variables } & & \multicolumn{2}{|c|}{ PCOs } & \multirow[t]{2}{*}{ p-VALUE } \\
\hline & & No & Yes & \\
\hline \multirow[t]{2}{*}{ Ovarian disease $^{4}$} & No (N\%) & $281(94.3)$ & $17(5.7)$ & $<0.001$ \\
\hline & Yes $(\mathrm{N} \%)$ & $63(75)$ & $21(25)$ & \\
\hline \multirow[t]{2}{*}{ Fields inherited ${ }^{4}$} & No (N\%) & $306(92.4)$ & $25(7.6)$ & 0.001 \\
\hline & Yes $(\mathrm{N} \%)$ & $41(77.4)$ & $12(22.6)$ & \\
\hline \multirow[t]{5}{*}{ Exercise $^{4}$} & Very low (N\%) & $151(89.9)$ & $17(10.1)$ & 0.86 \\
\hline & Low (N\%) & $50(87.7)$ & $7(12.3)$ & \\
\hline & Moderate (N\%) & $96(89.7)$ & $11(10.3)$ & \\
\hline & High (N\%) & $28(90.3)$ & $3(9.7)$ & \\
\hline & Very high (N\%) & $9(100)$ & $(0.0)$ & \\
\hline \multirow[t]{2}{*}{ Other diseases ${ }^{1}$} & No (N\%) & 307(91.1) & $30(8.9)$ & 0.081 \\
\hline & Yes $(\mathrm{N} \%)$ & $39(83)$ & $8(17)$ & \\
\hline \multirow[t]{3}{*}{ Medication4 } & Oral glucose-lowering Drug\% & 3.8 & 27.4 & $<0.001$ \\
\hline & Thyroid treatment drug\% & 31 & 27 & 0.1 \\
\hline & Other medication $\%$ & 5 & 7 & 0.2 \\
\hline \multirow[t]{2}{*}{$\operatorname{BMI}\left(\mathrm{kg} / \mathrm{m}^{2}\right) 2$} & $\operatorname{Mean}(\mathrm{SD})$ & $24.13(4.5)$ & $23.99(6.7)$ & 0.684 \\
\hline & $\mathrm{NO}=376$ & & & \\
\hline \multirow[t]{2}{*}{$\mathrm{AGE}^{2}$} & mean(SD) & $29.84(6.6)$ & $26.78(6.9)$ & 0.008 \\
\hline & $\mathrm{NO}=385$ & & & \\
\hline \multirow[t]{2}{*}{$\mathrm{Kcal}^{3}$} & mean(SD) & $6410(30781.9)$ & $4274(3673.2)$ & 0.94 \\
\hline & $\mathrm{NO}=381$ & & & \\
\hline Age at menarche ${ }^{2}$ & mean(SD) & $13.35(2)$ & $13.02(2.8)$ & 0.359 \\
\hline
\end{tabular}

1- Other disease: diabetes, thyroid disease 2-T-test 3-Mann -Whiteny Test 4-Chi-Square 
Considering the importance of family history, $22.6 \%$ of the patients with PCOS were shown to have a family history of PCOS. After adjusted logistic regression for medication, logistic regression analysis discovered that there was no relationship between genetics and PCOS $(\mathrm{p}=0.13)$, in contrast, a negative association was detected between the background of ovarian diseases $(\mathrm{OR}=0.2 \mathrm{CI}(95 \%)=0.082-0.490$; $\mathrm{P}<0.001)$, age $(p=0.012, \mathrm{OR}=0.9 \mathrm{CI}(95 \%)=0.787$ $0.934)$, as confounding variables and PCOS. Based on the results, BMI and PCOS are not significantly associated $(p$-value $=0.068)$.

However, no statistically important association was observed between the intake of LEUC, METH, CYS, PHE, VAL, ASP, GLUT, total protein and PCOS. Based on the results, the consumption of Met and PCOS were not significantly related but their relationship may be notable ( $\mathrm{p}$ value $=0.068)$ (table2) So that for every $1 \mathrm{mg}$ increase in methionine intake may lead to a decrease in PCOS disorder by $0.6 \%$. And every $1 \mathrm{mg}$ increase in the Glycine intake, leads to a \%2-decrease in the risk factors of PCOS. Conversely, every $1 \mathrm{mg}$ increase in the prolin consumption resulted in a $\% 6$ rise in the risk factors (table 2).

\section{Disc ussion}

In this study, the effect of amino acids and protein intake was assessed in patients with PCOS. In this study, after adjusted logistic regression for medication, significant connection was observed between glycine, prolin, and methionine with PCOS.

Although studies on the metabolic profiles of PCOS patients are limited to the plasma values (20). Some evidence has recommended that a diet with replacement of carbohydrates with protein has metabolic benefits in the treatment of polycystic ovary syndrome (PCOS) (21). Nonetheless, Azadbakht et al, revealed that increased red meat intake is related to inflammation risk (22).

Hemmings et al conducted a study to assess amino acid profiling in human oocytes with different competence to mature to metaphase II (MII) in vitro. The result showed that the reduction of key amino acids was connected to the maturation potential of human oocytes in vitro. Arrested oocytes at the germinal vesicle (GV) stage discharged, had significantly more valine and isoleucine than those which developed to metaphase I (MI) or metaphase II (MII) (23).

Table 2. The relationship between type of amino acid, total protein intake and other factors on risk of PCOSs

\begin{tabular}{llll}
\hline Variable & CI $(95 \%)$ & OR(adjusted $\left.{ }^{*}\right)$ & P-value \\
\hline MALT & $0.99-1$ & 0.99 & 0.096 \\
LEUC & $0.99-1$ & 0.99 & 0.14 \\
MET & $0.98-1$ & 0.99 & 0.06 \\
CYST & $0.99-1$ & 1 & 0.27 \\
PHE & $0.99-1$ & 0.99 & 0.13 \\
TYR & $1.001-1.003$ & 1 & 0.003 \\
VAL & $0.93-1$ & 0.96 & 0.083 \\
ARG & $1.001-1.01$ & 1 & 0.013 \\
ASP & $1-1.005$ & 1 & 0.098 \\
GLU & $0.992-1.041$ & 1 & 0.2 \\
GLY & $0.97-0.99$ & 0.98 & 0.01 \\
PRO & $1.003-1.073$ & 1.04 & 0.034 \\
BCAA/AAA & $0.98-0.99$ & 0.96 & 0.04 \\
KCAL** & $1.000-1.000$ & 1 & 0.083 \\
AGE & $0.85-0.98$ & 0.9 & 0.012 \\
Ovarian disease & $3.2-19.11 ?$ & 7.8 & 0.001 \\
BMI & $0.97-1.1$ & 1 & 0.19 \\
Fields inherited & $0.71-5.1$ & $2 ?$ & $2 ?$ \\
Other diseases & $0.65-6.1$ & 2 & 0.22 \\
Medication & $1.33-8.9$ & 3.4 & 0.11 \\
Age at menarche & $0.73-1$ & 0.87 & 0.1 \\
TOTAL PROTEIN & $0.96-1$ & 0.98 & 0.211 \\
\end{tabular}

\footnotetext{
*: OR was adjusted for drugs

$* *$ : as reference group
} 
Additionaly, the lack of balance amongst pro and anti-inflammatory cytokines and impaired glucose tolerance in the plasma, contributed to the pathophysiology of PCOS (24). It has also been shown that the levels of anti-inflammatory cytokines can be increased by glycine while reducing the values of inflammatory cytokines $(25,26,27)$, which may interrupt with steroidogenesis, follicular maturation and ovulation and thus support ovarian dysfunction $(28,29)$. Our result indicated that glycine has inverse relation to PCOS $(\mathrm{P}<0.5)$. Moreover, glycine treatment reduces the levels of pro-inflammatory cytokines and ameliorates glucose metabolism in T2DM (30,31). Therefore, glycine may be measured as the advantageous dietary factor for patients with inflammation disorders, such as PCOS subjects. In association with serine, it is probable that it applies to similar properties to glycine through transfiguration to glycine $(32,20)$.

Additionally, researchers observed that the amount of valine and tryptophan were generally raised in PCOS groups. Unlike that, the levels of glycine in four phenotypes of PCOS were reduced remarkably in comparison with the corresponding control group. Moreover, upper levels of alanine, serine, threonine, phenylalanine, ornithine and tyrosine were observed in ovulatory PCOS patients (37).

Also, in this study, it was found that BCAA/AAA ratio had inverse correlation to PCOS risk. Chun-mei Zhang accompanied a prospective study in PCOS subjects (20). The rate of aromatic amino acid (AAA) were augmented in PCOS participants, non-dependent to obesity, whereas the levels of branched-chain amino acid (BCAA), glutamic acid, phenylalanine, alanine, and arginine enhanced with body mass index regardless of the PCOS condition. This study showed that advanced levels of leucine, valine and glutamic acid were detected in insulin resistant PCOS group. Furthermore, serine and glycine levels were highly associated with PCOS patients $(\mathrm{r}=0.67)$.

Equally Carmina et al, had reported that the total levels of numerous amino acids was blocked in ovulatory women with PCOS in comparison with the control group, which amplified protein synthesis in women that lean muscle mass was augmented (34). But, the production of the considered amino acids: serine, threonine, phenylalanine, tyrosine and ornithine were raised only in the an ovulatory PCOS groups, which signified that increment of these five amino acids might be directly linked to ovulatory dysfunction through amplified ovarian uptake in PCOS patients (33).

The metabolic pathway associated with PCOS can be explained in this way. The afflicted metabolic pathways in PCOS patients involved the tricarboxylic acid (TCA) and other metabolic pathways. Also, circulating branched-chain amino acids and aromatic amino acids were connected to insulin resistance and diabetes mellitus in the prospective cohorts (33). Higher levels of peripheral glucose uptake and reduced hepatic lactate changes to glucose, proposed the enhanced glycolysis in muscle and prohibited gluconeogenesis in liver through existence of PCOS. Additionally, the PCOS patients were attended to have noticeable impairment of TCA cycle. This was revealed in the decreased citrate level and raised levels of plasma threonine, valine, phenylalanine and tyrosine, making a drop in succinyl-CoA and fumarate. Furthermore, the women with PCOS progressed quickly to a state of lipolysis and protein catabolism, via elevated values of plasma fatty acids and amino acids. An increased level of ornithine and diminished level of arginine guaranteed the unbalance of the urea cycle (34).

Tyrosine is an aromatic amino acid which is key in the synthesis of thyroid hormones, catecholamines, and melanin (35). Also, insulin commences its cellular responses by binding to the cellular receptor that contains insulin-stimulated tyrosine kinase activity. Amino acid such as glycine is involved in producing fumarat, pyruvate and succinyl coA. As these substances are essential factors in metabolism of carbohydrates, they can have an impact on insulin discharge (36).

The first limitations of this research was its crosssectional design which made it unable to find the cause-effect relations. The second limitation was not analyzing the hormonal balance like thyroid, prolactin and HOMA-IR (Homeostasis Model Assessment of Insulin Resistance) from the subjects. Inversely, the large number of the samples, lack of similar study in Iran directly addressing the connection between proteins and amino acids intake and PCOS, assessing the foods consumptions by means of a validated FFQ, using sonography test known as the most reliable method for PCOS diagnose were strength points of this study. 
More studies with more powerful designs are required to define the effect of different level of amino acids and protein intake on PCOS occurrence, symptoms and also treatment.

No conclusion?

\section{Financial disclosure}

The authors declared no financial interest.

\section{Funding/Support}

This work was financially supported by Isfahan University of Medical Science

\section{References}

1. Rajaeieh G, Marasi M, Zahra Shahshahan, Fatemmeh Hassanbeigi F, Safavi SM. The Relationship between Intake of Dairy Products and Polycystic Ovary Syndrome in Women Who Referred to Isfahan University of Medical Science Clinics in 2013. Int J Prev Med 2014; 6: 687-694.

2. Chavarro JE, Toth TL, Wright DL, Meeker JD, Hauser R. Body mass index in relation to semen quality, sperm DNA integrity, and serum reproductive hormone levels among men attending an infertility clinic. Fertil Steril. 2010 May 1;93(7):2222-31

3. Gambineri A, Patton L, Vaccina A, Cacciari M, MorselliLabate AM, Cavazza C, et al. Treatment with flutamide, metformin, and their combination added to a hypocaloric diet in overweight-obese women with polycystic ovary syndrome: a randomized, 12-month, placebo-controlled study. J. Clin. Endocrinol. Metab 2006; 10: 3970-3980.

4. Le Donne M, Alibrandi A, Giarrusso R, Lo Monaco I, Muraca U, Ginecologica M. Diet, metformin and inositol in overweight and obese women with polycystic ovary syndrome: Effects on body composition. Minerva Ginecol 2012; $1: 23-29$.

5. Pasquali R, Gambineri A, Cavazza C, Ibarra Gasparini D, Ciampaglia W, Cognigni GE, et al. Heterogeneity in the responsiveness to long-term lifestyle intervention and predictability in obese women with polycystic ovary syndrome. Eur J Endocrinol 2011; 1:53-60.

6. Artimani T, Saidijam M, Aflatoonian R, Amiri I, Ashrafi $\mathrm{M}$, Shabab N, et al. Estrogen and progesterone receptor subtype expression in granulosa cells from women with polycystic ovary syndrome. Gynecol Endocrinol 2015; 5: 379-383.

7. Norman RJ, Dewailly D, Legro RS, Hickey T E. Polycystic ovary syndrome. The Lancet 2007; 370: 685-697.

8. Pirak A, Kashani ZA, Azarkish F, Salehian T, Hagani H, Parsania Z, et al. A study on correlation between gestational diabetes and history polycystic ovary syndrome in women referred to Akbar Abady Hospital in Tehran 1998-2005.Sci J Ilam Univ Med Sci 2009; 4:17-25

9. Motta $\mathrm{AB}$. The role of obesity in the development of polycystic ovary syndrome. Curr Pharm Des 2012; 10:24822491.

10. Kachoie A, Jahanipoor A, Ghahiri A. Evaluation of serum prolactin levels in patients with polycystic ovary syndrome. J Isfahan Med Sch 2012; 182: 353-358.
11. Barba G, Russo P. Dairy foods, dietary calcium and obesity: A short review of the evidence. Nutr Metab Cardiovasc Dis 2006; 6: 445-451.

12. Hajishafieha M. Causes and treatment of chronic anovulation: Institute Publications, Witnessed and Veterans; 2003.

13. Salmabadi Z, Mohseni-Kouchesfahani H, Parivar K, Karimzadeh L. Effect of grape seed extract on lipid profile and expression of interleukin-6 in polycystic ovarian syndrome wistar rat model. Int J Fertil Steril 2017; 3: 176183.

14. Salih SM., Salama SA, Fadl AA, Nagamani M, Al-Hendy A. Expression and cyclic variations of catechol-O-methyl transferase in human endometrial stroma. Int $\mathrm{J}$ Fertil Steril 2008; 3: 789-797.

15. Szczuko M, Zapałowska-Chwyć M, Maciejewska D, Drozd A, Starczewski A, Stachowska E. High glycemic index diet in PCOS patients. The analysis of IGF I and TNF- $\alpha$ pathways in metabolic disorders. Med. hypotheses 2016: 42-47.

16. Ahmadi A, Akbarzadeh M, Mohammadi F, Akbari M, Jafari B, Tolide-Ie H. Anthropometric characteristics and dietary pattern of women with polycystic ovary syndrome. Indian J Endocrinol Metab 2013; 4: 672-676.

17. Oono T, Yamasaki T, Zaitsu J, Saeki I, Iwamoto T, Harima Y, et al. Association of tyrosine with insulin resistance in hepatitis C virus-related chronic liver disease. Hepatol Res 2014; 10: E54-E62

18. Noakes M, Keogh JB, Foster PR, Clifton PM. Effect of an energy- restricted, high- protein, low-fat diet relative to a conventional high- carbohydrate, low-fat diet on weight loss, body composition, nutritional status, and markers of cardiovascular health in obese women. Am J Clin Nutr 2005; 6:1298-1306.

19. Hosseini-Esfahani F, Asghari G, Mirmiran P, Farahani PJ, Azizi F. Reproducibility and relative validity of food group intake in a food frequency questionnaire developed for the theran lipid and glucose study. Razi J Med Sci 2010; 6: 41-55.

20. Zhang C, Zhao Y, Li R, Yu Y, Yan L, Li L, et al. Metabolic heterogeneity of follicular amino acids in polycystic ovary syndrome is affected by obesity and related to pregnancy outcome. Zhang et al. BMC Pregnancy Childbirth 2014; 11: $1-7$

21. Sørensen LB1, Søe M, Halkier KH, Stigsby B, Astrup A. Effects of increased dietary protein-to-carbohydrate ratios in women with polycystic ovary syndrome. Am J Clin Nutr 2012; $1: 39-48$

22. Azadbakht L, Esmaillzadeh A. Red meat intake is associated with metabolic syndrome and the plasma C-reactive protein concentration in women. J Nutr 2008; 2: 335-339.

23. Kelly CC, Lyall H, Petrie JR, Gould GW, Connell JM, Sattar $\mathrm{N}$. Low grade chronic inflammation in women with polycystic ovarian syndrome. J Clin Endocrinol Metab 2001; 6: 24532455.

24. Amato G, Conte M, Mazziotti G, Lalli E, Vitolo G, Tucker AT, et al. Serum and follicular fluid cytokines in polycystic ovary syndrome during stimulated cycles. Obstet Gynecol 2003; 6: 1177-1182.

25. Garcia-Macedo R, Sanchez-Munoz F, Almanza-Perez JC, Duran-Reyes G, Alarcon-Aguilar F, Cruz M. Glycine increases mRNA adiponectin and diminishes proinflammatory adipokines expression in 3 T3-L1 cells. Eur J Pharmacol 2008; 3: 317-321. 
26. Spittler A, Reissner CM, Oehler R, Gornikiewicz A, Gruenberger T, Manhart N, et al. Immunomodulatory effects of unit on LPS-treated monocytes: reduced TNF-alpha production and accelerated IL-10 expression. FASEB J 1999; 3: $563-571$

27. Alarcon-Aguilar FJ, Almanza-Perez J, Blancas G, Angeles S, Garcia-Macedo R, Roman R, et al. Glycine regulates the production of pro-inflammatory cytokines in lean and monosodium glutamate-obese mice. Eur J Pharmacol 2008; 3: $152-158$

28. Montgomery-Rice V, Limback SD, Roby KF, Terranova PF. Differential responses of granulosa cells from small and large follicles to follicle stimulating hormone (FSH) during the menstrual cycle and acyclicity: effects of tumour necrosis factor-alpha. Hum Reprod 1998; 5: 1285-1291.

29. Vural P, Degirmencioglu S, Saral NY, Akgul C. Tumor necrosis factor alpha (-308), interleukin-6 (-174) and interleukin-10 (-1082) gene polymorphisms in polycystic ovary syndrome. Eur J Obstet Gynecol Reprod Biol 2010; 1: 61-65.

30. Cruz M, Maldonado-Bernal C, Mondragon-Gonzalez $\mathrm{R}$, Sanchez-Barrera R, Wacher NH, Carvajal-Sandoval G, et al. Glycine treatment decreases proinflammatory cytokines and increases interferon-gamma in patients with type 2 diabetes. J Endocrinol Invest 2008; 8: 694-699.
31. Sekhar RV, McKay SV, Patel SG, Guthikonda AP, Reddy VT, Balasubramanyam A, et al. Glutathione synthesis is diminished in patients with uncontrolled diabetes and restored by dietary supplementation with cysteine and glycine. Diabetes Care 2011; 1: 162-167.

32. Kikuchi G, Hiraga K. The mitochondrial glycine cleavage system. Unique features of the glycine decarboxylation. Mol Cell Biochem 1982; 3: 137-149

33. Zhao Y, Li F, Li R, Wang L, Yan Yang, Liu N,et al. Metabolic profiles characterizing different phenotypes of polycystic ovary syndrome: plasma metabolomics analysis. BMC Med 2012; 10:153

34. Carmina E, Guastella E, Longo RA, Rini GB, Lobo RA. Correlates of increased lean muscle mass in women with polycystic ovary syndrome. Eur J of endocrinol 2009; 583589

35. Murray, Robert K, Peter L. Gross. "Harper's Illustrated Biochemistry, 2012.

36. Chen Z, Gibson TB, Robinson F, Silvestro L, Pearson G, Xu B, et al. MAP kinases. Chem rev 2009; 8: 2449-2476. 